\title{
Medial Temporal Lobe Activations in fMRI and PET Studies of Episodic Encoding and Retrieval
}

\author{
Daniel L. Schacter ${ }^{1 *}$ and Anthony D. Wagner ${ }^{1,2}$ \\ ${ }^{1}$ Department of Psychology, Harvard University, \\ Cambridge, Massachusetts \\ ${ }^{2}$ Massachusetts General Hospital NMR Center, \\ Harvard Medical School, Boston, Massachusetts
}

\begin{abstract}
Early neuroimaging studies often failed to obtain evidence of medial temporal lobe (MTL) activation during episodic encoding or retrieval, but a growing number of studies using functional magnetic resonance imaging (fMRI) and positron emission tomography (PET) have provided such evidence. We review data from fMRI studies that converge on the conclusion that posterior MTL is associated with episodic encoding; too few fMRI studies of retrieval have reported MTL activations to allow firm conclusions about their exact locations. We then turn to a recent meta-analysis of PET studies (Lepage et al., Hippocampus 1998;8:313322 ) that appears to contradict the fMRI encoding data. Based on their analysis of the rostrocaudal distribution of activations reported during episodic encoding or retrieval, Lepage et al. (1998) concluded that anterior MTL is strongly associated with episodic encoding, whereas posterior MTL is strongly associated with episodic retrieval. After considering the evidence reviewed by Lepage et al. (1998) along with additional studies, we conclude that PET studies of encoding reveal both anterior and posterior MTL activations. These observations indicate that the contradiction between fMRI and PET studies of encoding was more apparent than real. However, PET studies have reported anterior MTL encoding activations more frequently than have fMRI studies. We consider possible sources of these differences. Hippocampus 1999;9:7-24.

(1) 1999 Wiley-Liss, Inc.
\end{abstract}

KEY WO RDS: functional neuroimaging; PET; fMRI; encoding; retrieval; medial temporal lobes

\section{INTRODUCTION}

Understanding the mnemonic functions of the hippocampus and related medial temporal lobe (MTL) structures has been advanced greatly by the study of brain-damaged patients and experimental animals. The recent availability of such neuroimaging techniques as positron emission tomography (PET) and functional magnetic resonance imaging (FM RI) raises the exciting possibility of examining hippocampal/M TL functions in healthy individuals with intact memory. However, several early neuroimaging

Grant sponsor: H uman Frontiers Science Program; Grant number: RG 0126; Grant sponsor: National Institute on Aging; Grant numbers: AG08441, AG05778; Grant sponsor: National Institute of Mental Health; Grant number: MH57915.

*Correspondence to: Daniel L. Schacter, Department of Psychology, Harvard U niversity, 33 Kirkland St. Cambridge, MA 02138.

E-mail:dls@wjh.harvard.edu

Accepted for publication 310 ctober 1998 studies of encoding or retrieval failed to provideevidence of hippocampal or related MTL activations (e.g., Kapur et al., 1994; Shallice et al., 1994; Tulving et al., 1994; Buckner et al., 1995). These negative findings have led to various explanations for the unexpected absence of $M T L$ activity. Thus, it has been suggested that MTL structures may be continuously active and, therefore, often missed by standard subtraction techniques; they may not show sufficiently robust changes in blood flow across experimental conditions to be detected by PET and $\mathrm{fMRI}$; or they may be involved in consolidation processes that are not as readily imaged as are encoding and retrieval processes (compare Fletcher et al., 1995; Haxby, 1996; M artin et al., 1997; Fernandez et al., 1998). In contrast to these early negative findings, more recent $\mathrm{FM} R \mathrm{R}$ and PET studies have provided convincing evidence that M TL activity is modulated during encoding and retrieval (e.g., Schacter et al., 1995, 1996a, 1997b; N yberg et al., 1996; O wen et al., 1996; Stern et al., 1996; Dolan and Fletcher, 1997; Gabrieli et al., 1997; H enke et al., 1997; M artin et al., 1997; Rombouts et al., 1997; Rugg et al., 1997; Brewer et al., 1998; Fernandez et al., 1998; M aguire et al., 1998a,b; Wagner et al., 1998).

In view of the increasing numbers of positive observations of M TL activity during encoding and retrieval, it is now possible to begin to ask of neuroimaging data theoretically interesting questions concerning the nature of M T L involvement in memory functions. O ne fundamental question that is potentially addressable with neuroimaging techniques concerns the role of specific $M T L$ regions in encoding and retrieval processes: Are the same MTL regions involved in encoding and retrieval, or do different regions within the MTL contribute differentially to encoding and retrieval? A second issue that needs to beaddressed concerns whether findings from $\mathrm{FMRI}$ and PET studies are similar or different, and in what ways. $\mathrm{On}$ the onehand, if the two techniques yield similar patterns of results, there is reason to have increased confidence about the generality of the conclusions that can be drawn from either technique alone. $0 \mathrm{n}$ the other hand, if different patterns 
of results are observed in $\mathrm{FMRI}$ and PET studies, it becomes important to attempt to understand the nature and origins of those differences.

FMRI and PET are similar techniques in the sense that both rely on changes in hemodynamic responses that are correlated with changes in neuronal activity: $\mathrm{fM} \mathrm{RI}$ is sensitive to oxygenationlevel-dependent changes in the magnetic properties of blood (BOLD), and PET is sensitive to local changes in blood flow. However, the two techniques differ in many ways (Xiong et al., 1998) and it is possible that they may thus provide different insights into brain activity. Although few direct comparisons have been reported (e.g., Xiong et al., 1998), PET and fM RI studies of memory have yielded a number of notable similarities (e.g., O jemann et al., 1998; for reviews, see Ungerleider, 1995; C abeza and Nyberg, 1997; Buckner and Koutstaal, 1998; Schacter and Buckner, 1998).

In this paper we first review evidence from $\mathrm{FMRI}$ studies concerning the nature of MTL activations during encoding and retrieval. Although only a few fMRI studies have reported MTL activations during retrieval, data from a variety of $\mathrm{FMRI}$ studies converge on the idea that posterior MTL regions, mainly in the parahippocampal gyrus, play an important role in encoding processes.

$\mathrm{N}$ ext, we compare findings from $\mathrm{fM} R \mathrm{R}$ studies to PET studies of encoding and retrieval. Lepage et al. (1998) recently reviewed PET studies that have revealed significant activations in the hippocampus and related MTL structures during encoding or retrieval. They noted a striking and unexpected regularity in the distribution of activations along the rostrocaudal axis: encoding activations tended to be clustered in more rostral (i.e., anterior) $M T L$ regions whereas retrieval activations tended to be clustered in more caudal (i.e., posterior) MTL regions (referred to as the hippocampal encoding/retrieval pattern or "H IPER"). Thus, the pattern for PET encoding activations appears to be precisely the opposite of the pattern we note for $\mathrm{fMRI}$ encoding activations. These contrasting patterns of results raise the possibility that PET and $\mathrm{fM} \mathrm{RI}$ are providing fundamentally different pictures of $\mathrm{MTL}$ activity. Although the matter cannot be settled definitively at the present time, after discussing in some detail Lepage et al.'s (1998) analysis of PET results we suggest that the PET and $\mathrm{FMRI}$ data concerning M TL encoding activations are not contradictory. We then consider possible reasons for some of the differences that do exist between PET and fM RI studies.

\section{FMRI STUDIES OF ENCODING} AND RETRIEVAL

\section{Encoding Studies}

M ost fMRI evidence of MTL activation has been obtained from experiments concerned with memory encoding processes. In one of the first such studies, Stern et al. (1996) showed participants colored magazine pictures of everyday scenes. In the experimental condition, participants viewed 40 pictures, displayed at a rate of one every three seconds, and were instructed to "view the pictures carefully so that they could recognize them later" (Stern et al., 1996, p. 8661). D uring the control condition, participants viewed a single picture that was presented repeatedly at the same rate as the experimental pictures. Stern et al. (1996) reported activation of the posterior aspects of the hippocampus and the parahippocampal gyrus during the experimental condition compared to the control condition. Brain atlas co-ordinates (Talairach and Tournoux, 1988) for significant M TL activations in the Stern et al. (1996) experiment, along with co-ordinates for M TL activations from all other $\mathrm{FM} \mathrm{RI}$ studies that we review and that provided co-ordinates, are presented in Table 1.

Gabrieli et al. (1997) reported evidence that is consistent with the results from Stern et al.'s (1996) study. In their incidental encoding task, participants saw 12 sets of eight pictures of everyday scenes; each set included four indoor and four outdoor scenes, which were presented for three seconds each. Subjects made indoor/outdoor scene judgments for each picture, and were told to try to remember the pictures. Participants alternated between conditions in which pictures were novel (i.e., shown for the first time) or familiar (i.e., the same two pictures were shown repeatedly). Compared to encoding of familiar pictures, encoding of novel pictures was associated with posterior MTL activation situated bilaterally in the parahippocampal cortex.

A study by Rombouts et al. (1997) yielded similar results. During an encoding condition, participants memorized pictures of scenes that each contained two objects, with the names of the objects written directly below the picture. Compared to a control condition wherethe same scene was presented repeatedly, the encoding condition produced activation of posterior M TL, in parahippocampal cortex, for eleven of twelve subjects. In addition, hippocampal activation was observed in seven of thetwelve subjects.

W hile the findings of Stern et al. (1996), G abrieli et al. (1997), and Rombouts et al. (1997) all suggest a link between posterior MTL activity and encoding processes, the results of these studies leave open at least three issues. First, it is possible that the observed M TL activations reflect responses to novel stimuli. In each of the studies, novel pictures were compared to repeated pictures, and previous research with PET has implicated the parahippocampal gyrus in response to novelty (e.g., Tulving et al., 1996; Elliot and Dolan, 1998; these "novelty effects" also could be interpreted as activation reductions that are neural correlates of repetition priming - for discussion, see Schacter and Buckner, 1998; W iggs and $M$ artin, 1998). Second, in all three experiments (as in most PET and fM RI studies), items from different conditions were pre sented in separate "blocks," with analysis of fM RI responses reflecting averaged activity in one block compared to another. In such blockeddesign studies, it is possible that differences between conditions reflect general differences in strategies that subjects use in one block of trials compared to another, rather than trial-by-trial differences in encoding operations that determine the subsequent memorability of specific target stimuli. Third, because complex objects and visuo-spatial scenes were used in these studies (see also Aguirre et al., 1998; Epstein and Kanwisher, 1998), the observed posterior M TL activations may be specific to this type of material (Lepage et al., 1998). Several more recent studies have begun to address these issues.

Fernandez et al. (1998) reported an experiment in which participants studied lists of common words. They used a blocked 
TABLE 1.

Stereotaxic Co-Ordinates of Encoding and Retrieval Activations From fMRI Studies*

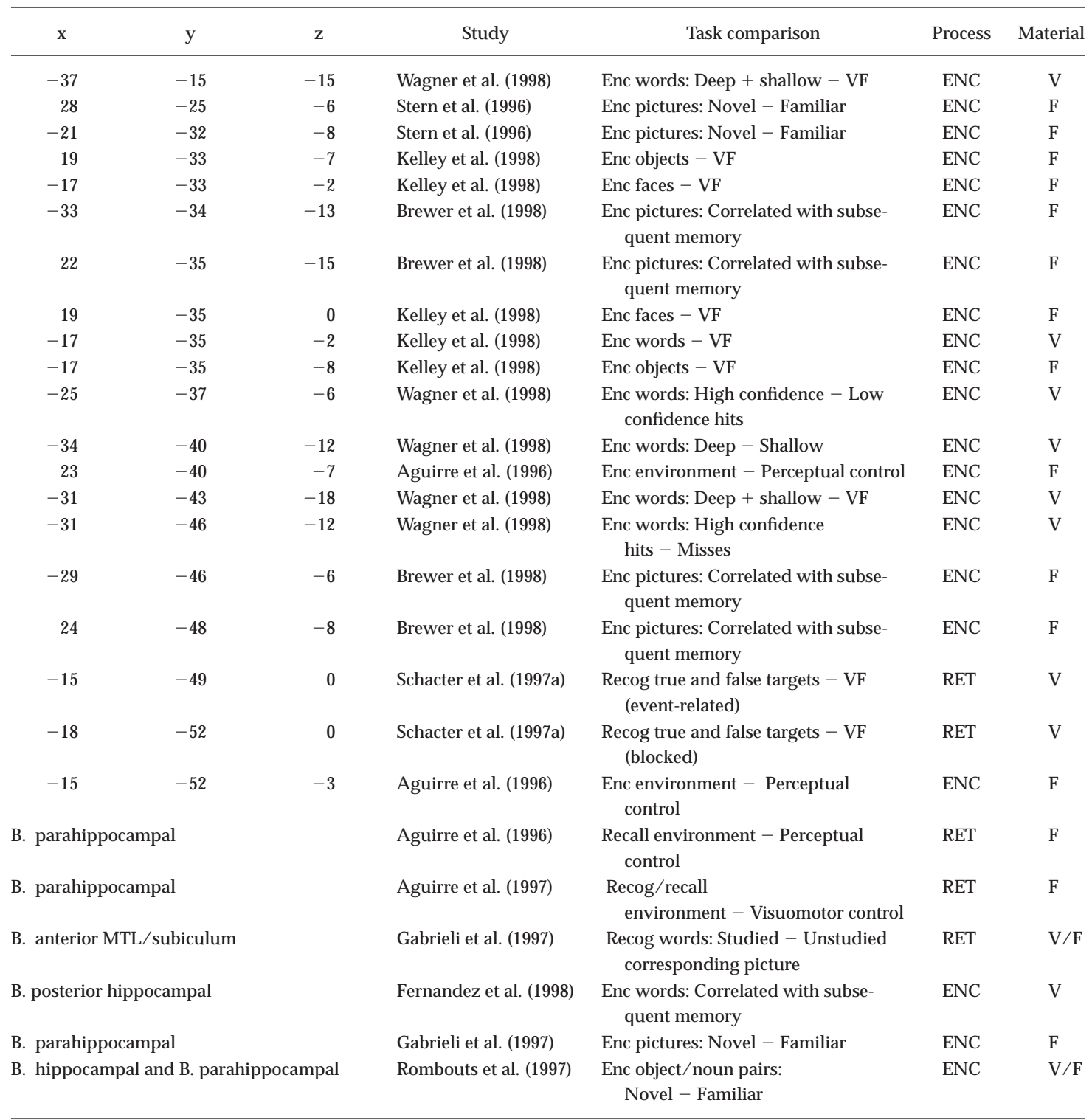

*Stereotaxic co-ordinates (xyz) from the brain atlas of Talairach and Tournoux (1988); for comparisons in which co-ordinates were not provided, we provide the authors' reported MTL location. $\mathrm{B}=$ bilateral; ENC = encoding; $\mathrm{F}=$ figural materials; Recog $=$ recognition; $\mathrm{RET}=$ retrieval; $\mathrm{V}=$ verbal materials; $\mathrm{VF}=$ visual fixation.

design in which 15 words were studied during scanning, followed by a brief period of distraction and then a free recall test in which participants tried to remember words from the preceding list. There were 20 repetitions of this study-distraction-test cycle, each time with a new set of target words. Fernandez et al. (1998) analyzed their data by correlating the magnitude of $\mathrm{fM}$ RI signals during five-word encoding blocks with the number of words from each block that were subsequently produced on the free recall test. For 11 of 13 participants, they found significant positive correlations between the number of recalled words and signal intensity in posterior hippocampal/M TL regions.

TheFernandez et al. (1998) study suggests that M TL responses during encoding extend beyond the domain of visuo-spatial materials and may involve processes other than, or in addition to, novelty detection. Even stronger evidence on these points is provided by two recent studies that used "event-related" fM RI methodologies. As noted earlier, most fM RI and PET studies have used blocked designs, where items from different experimental conditions are grouped together during encoding or retrieval. By contrast, in event-related fMRI studies, items from different experimental conditions can be randomly intermixed during encoding or retrieval, as is typically done in purely cognitive/ behavioral studies. Responses can be sorted later according to whether or not an item is successfully remembered, much in the manner that data from studies using electrophysiological measures, such as event-related potentials (ERPs), can be selectively 
sorted and averaged (for discussion of event-related fM RI methods, see Buckner et al., 1996; Aguirre et al., 1997; Dale and Buckner, 1997; Josephs et al., 1997; Rosen et al., 1998).

We recently reported two experiments, one using a blocked design and one using event-related $\mathrm{FMRI}$, to examine verbal encoding processes (Wagner et al., 1998). In the blocked-design experiment, blocks of 20 words (one-half denoting concrete entities, one-half abstract) were presented visually at a rate of one word every two seconds. Participants made semantic encoding judgments during half of the word blocks (they decided whether each word was abstract or concrete) and nonsemantic encoding judgments during the other half (deciding whether each word was presented in upper or lowercase). As expected from numerous previous studies (e.g., Craik and Lockhart, 1972), subsequent memory performance (as assessed by an old/new recognition test) was significantly higher for semantic blocks than for nonsemantic blocks. Analysis of fM RI data showed significantly greater activation in the left parahippocampal and fusiform gyri during semantic encoding than during nonsemantic encoding (several areas within the left inferior prefrontal cortex also showed greater activity during semantic than nonsemantic encoding; compare Kapur et al., 1994; Demb et al., 1995). A less constrained comparison to a fixation control also revealed left parahippocampal/ fusiform activation, and in addition yielded a more anterior hippocampal activation. Although we have listed this latter activation in Table 1, the finding must be treated cautiously, because (1) this anterior MTL region did not demonstrate differential activation in the more telling comparison of semantic and nonsemantic encoding conditions, and (2) the anterior MTL activation was not replicated in a second experiment.

The second experiment used event-related $\mathrm{FM} \mathrm{RI}$ to determine whether left parahippocampal (and prefrontal) responses to individual words during encoding predict subsequent memory performance, even under conditions in which depth of encoding is not manipulated experimentally. D uring each of six scans, 80 word trials and 40 visual fixation trials (i.e., participants looked at a crosshair) were randomly intermixed and presented at a rate of two seconds per trial. For word trials, participants decided whether each word was abstract or concrete. Approximately 20 minutes after the final scan was completed, participants were given a (nonscanned) recognition test on which they made old/new judgments about studied and nonstudied words; when they judged that a word was "old," they also indicated whether their judgment was accompanied by high confidence or low confidence. Event-related procedures allowed us to analyze the fM RI data by sorting encoding trials according to whether a word was subsequently remembered or forgotten. When we compared high confidence hits (i.e., "old" responses to studied words accompanied by high confidence) to misses (i.e., "new" responses to studied words), we found significant activation in the left parahippocampal and fusiform gyri; several left inferior prefrontal regions also showed significant activation.

To examine whether the effect could be attributed to time-ontask effects (e.g., subjects might have attended longer to words that were subsequently remembered than to words that were subsequently forgotten), a subset of the trials was selected such that response latencies during encoding were matched for subsequently remembered and forgotten items. This analysis revealed the same pattern of greater parahippocampal and prefrontal response for high confidence hits than for misses as seen in the overall analysis. A further analysis (not described in the short report of Wagner et al., 1998) that compared high confidence hits to low confidencehits again reveal ed significant left parahippocampal, fusiform, and prefrontal activations.

The overall pattern of results indicates that parahippocampal activations during processing of verbal experiences involve encoding mechanisms that extend beyond the domain of novelty detection, reflecting trial-by-trial differences in the efficacy of encoding operations rather than more general shifts in attention or strategy that could be operative in blocked-design experiments. Similar conclusions emerge from a complementary study by Brewer et al. (1998) that used event-related fM RI to examine encoding of nonverbal experiences. Participants in their experiments were shown 24 pictures of indoor and outdoor scenes (the same as those used by Gabrieli et al., 1997) during each of four scans, and judged whether each picture depicted an indoor or outdoor scene. Thirty minutes later, subjects were given a (nonscanned) recognition test on which they made old/new judgments about previously studied pictures and new pictures. For pictures judged as "old," participants also indicated whether they possessed a specific recollection of having seen the picture earlier (a "remember" judgment) or whether the picture just seemed familiar to them (a "know" judgment; see Gardiner and Java, 1993; Tulving, 1985). Brewer et al. (1998) found that activity level in parahippocampal cortex bilaterally (and in right inferior frontal gyrus) predicted subsequent remembering and forgetting. There was greater parahippocampal activity during encoding for pictures that were "remembered" specifically than for pictures that just seemed familiar, and also greater parahippocampal activity for familiar pictures compared to forgotten pictures. $^{1}$

The combined findings of Wagner et al. (1998) and Brewer et al. (1998) using event-related fM RI indicate some lateralization of posterior M TL responses during encoding: Wagner et al. (1998) found that left parahippocampal activity predicted word recognition, whereas Brewer et al. (1998) found that bilateral parahippocampal activity predicted picture recognition. Consistent with these observations, Kelley et al. (1998) reported a similar pattern of lateralization within each of two experiments that used blocked designs. In both experiments, subjects participated in three different encoding tasks in which they tried to memorize a series of words, nameable objects, or unfamiliar faces. Compared to a fixation control (Experiment 1) or a passive viewing control in which subjects simply looked at words, objects, or faces (Experiment 2), the intentional encoding conditions produced a signifi-

${ }^{1}$ Brewer et al. (1998) reported six parahippocampal foci that predict levels of subsequent memory. In Table 1, we have included four representative foci from left and right parahippocampal cortices (the two additional foci reported by Brewer et al. are: -34 $-29-18 ;-27-40-9$ ). It also should be noted that Brewer et al. did not sample from anterior MTL regions. 
cant left posterior MTL activation for words, and a significant bilateral posterior MTL activation for objects and faces. Direct comparisons between the two hemispheres revealed left>right activation for words and right>left activation for faces.

Whereas the previous studies all examined encoding of isolated words, objects, scenes, or faces, Aguirre et al. (1996) examined encoding of a complex spatial environment. In the encoding condition, participants actively explored the complex environment in order to remember it for a later test; in the control condition, they continually moved through a sparse circular corridor. Aguirreet al. (1996) reported activation of parahippocampal cortex bilaterally in the encoding condition compared to the control condition.

\section{Retrieval Studies}

O nly a handful of $\mathrm{fM} R \mathrm{RI}$ experiments have provided evidence of MTL activations during memory retrieval. In addition to the experiment involving encoding scans described earlier, $G$ abrieli et al. (1997) also scanned subjects after they had studied line drawings of objects. Subjects were shown words and were asked to recall whether they had seen a drawing with that name. In one condition, most of the words were the names of previously studied drawings and in another condition most of the words were names of drawings that had not been presented. Retrieval of previously studied drawings was associated with an anterior M TL activation in the vicinity of the subiculum. In an attempt to increase the similarity between this retrieval study and the previously described picture encoding study, Gabrieli et al. (1997) ran an additional two subjects on a retrieval task in which pictures were presented and subjects attempted to recall previously studied words. They found greater activation in the subiculum during a retrieval condition in which pictures primarily corresponded to previously studied words compared to a retrieval condition in which pictures corresponded mainly to nonstudied words.

In contrast to these anterior MTL retrieval activations, three other studies have reported evidence of more posterior MTL activations during retrieval. In two related experiments (one using a blocked design, the other an event-related design), Schacter et al. (1997a) exposed participants to lists of semantically associated words. Subjects were then scanned as they made old/new recognition judgments about previously studied words or related lures (i.e., "false targets") that were semantic associates of the previously studied words but had not actually been presented during the study trials. Compared to a fixation control condition, recognition of previously studied words and false targets was associated with activation in the left parahippocampal cortex.

Aguirre et al. (1996) examined retrieval of a recently learned complex environment (as described earlier). Similar to their findings concerning encoding of the environment, Aguirre et al. (1996) reported bilateral parahippocampal activation during retrieval. Aguirre and D'Esposito (1997) scanned subjects while they attempted to recall different aspects of a "virtual town" that they had become familiar with 2 to 3 days prior to scanning. In the appearance condition, participants were shown a scene from the town and asked to indicate whether it matched a name that was provided. In the position condition, the same stimuli were presented, followed by an arrow and the name of another target location; participants were required to indicate the direction of the other target relative to the presented target. Compared to a control condition where subjects viewed scrambled visual scenes, there was significant bilateral parahippocampal activation in both experimental conditions.

\section{SUMMARY OF FMRI FINDINGS}

In summary, the findings from $\mathrm{fM} R \mathrm{RI}$ studies converge on the observation that posterior regions of the M TL, involving mainly the parahippocampal gyrus and caudal aspects of the hippocampus, play an important role in memory encoding processes. This finding has been obtained in studies using verbal materials (Fernandez et al., 1998; Wagner et al., 1998), nonverbal materials (Aguirre et al., 1996; Stern et al., 1996; Aguirre and D 'Esposito, 1997; Gabrieli et al., 1997; Brewer et al., 1998), or both (Rombouts et al., 1997; Kelley et al., 1998); with a variety of analysis strategies, including subtraction (Aguirre et al., 1996; Stern et al., 1996; A guirre and D 'Esposito, 1997; Gabrieli et al., 1997; Rombouts et al., 1997; Kelley et al., 1998; Wagner et al., 1998), correlational techniques (Fernandez et al., 1998), and event-related procedures (Brewer et al., 1998; Wagner et al., 1998); and both when novelty detection processes are possible contributors to the observed activations (Stern et al., 1996; Gabrieli et al., 1997; Rombouts et al., 1997) and when they are not (Aguirre et al., 1996; A guirre and D 'Esposito, 1997; Brewer et al., 1998; Fernandez et al., 1998; K elley et al., 1998; Wagner et al., 1998). Although some studies have also produced evidence of more anterior M TL activation during encoding (Stern et al., 1996; Rombouts et al., 1997; Wagner et al., 1998), all of the reviewed studies reported posterior M T L activation during encoding. By contrast, too few fM RI data are available concerning M TL activations during retrieval in order to permit any firm conclusions about their rostrocaudal location (for a schematic depiction of all encoding and retrieval foci, see Figure 1).

\section{PET STUDIES OF ENCODING AND RETRIEVAL}

The data from $\mathrm{fM} R \mathrm{RI}$ studies of encoding appear compelling and consistent. It thus may be surprising that Lepage et al. (1998) recently reported a meta-analysis of PET studies that appears to yield a different and perhaps opposite conclusion from the $\mathrm{fM}$ R I studies. Lepage et al. (1998) summarized results from a database of 52 PET studies that obtained evidence of 54 individual MTL activations during encoding or retrieval. They noted a highly consistent pattern of findings: M TL activations during encoding tended to fall in the rostral portions of the rostrocaudal axis, 
TABLE 2.

Stereotaxic Co-Ordinates of Encoding and Retrieval Activations From PET Studies, Modified From Lepage et al. (1998)*

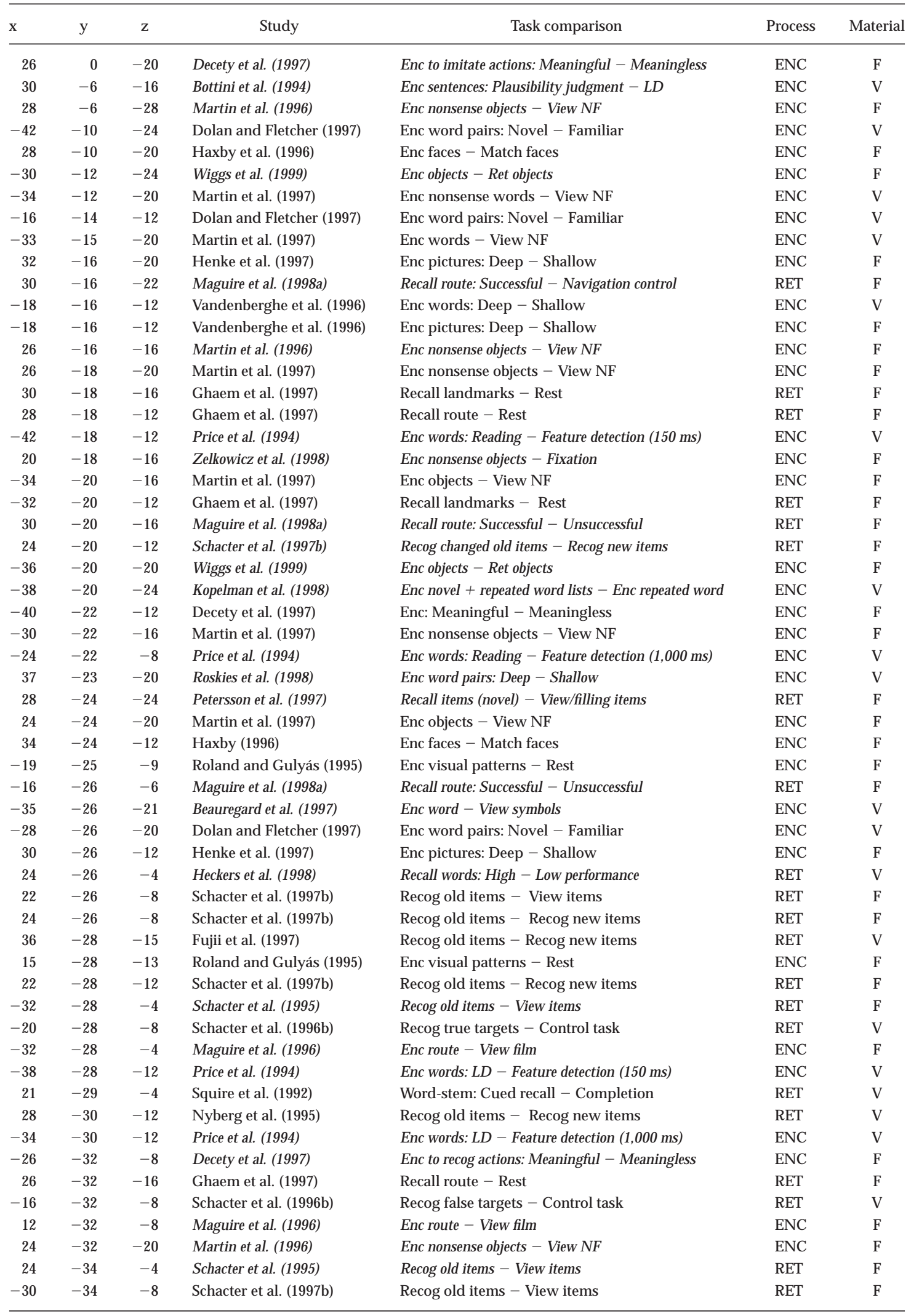


TABLE 2.

(Continued)

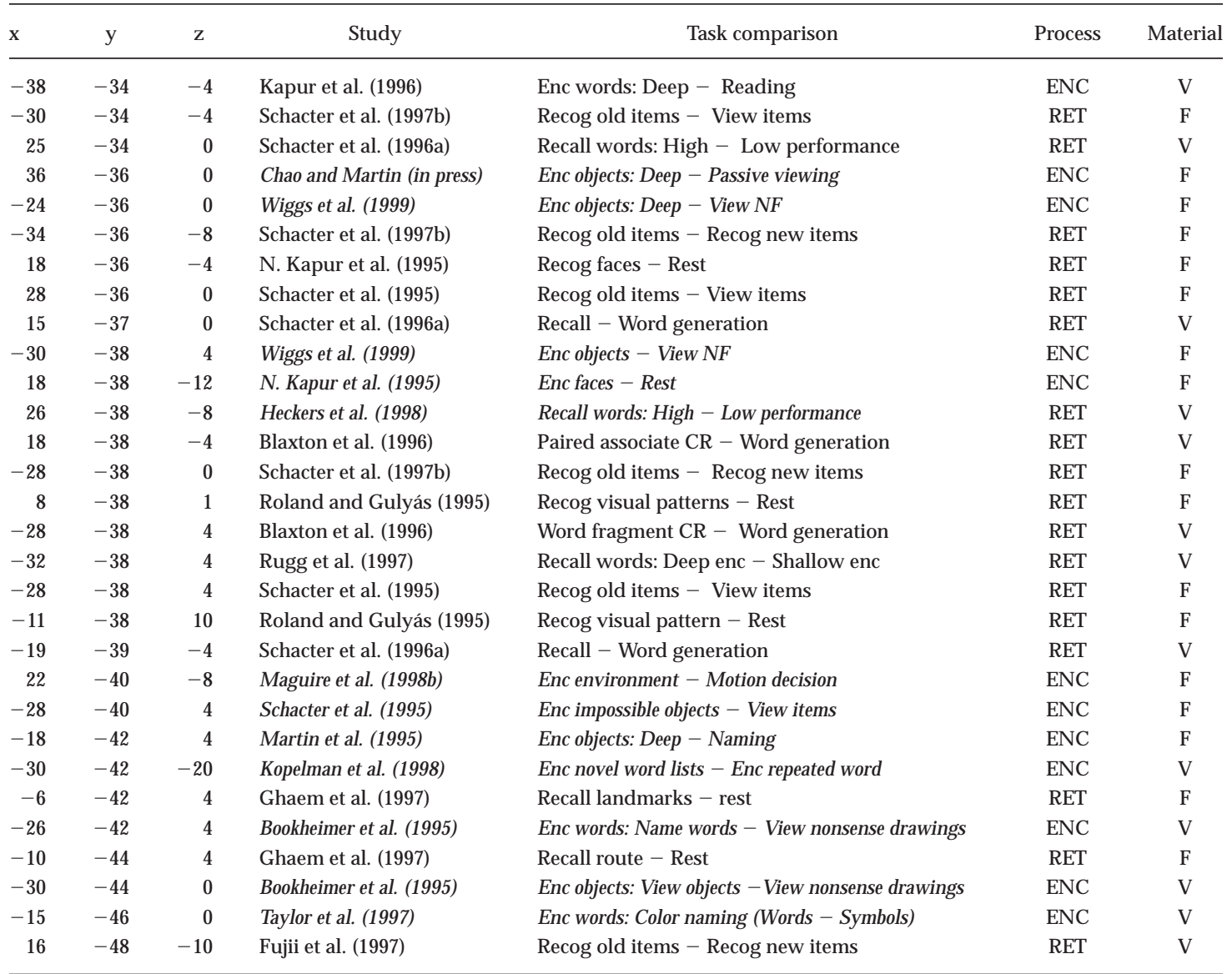

*Studies in italics did not appear in Lepage et al. (1998); see text for inclusion criteria. CR = cued-recall; ENC = encoding; F = figural materials; $\mathrm{LD}=$ lexical decision; $\mathrm{NF}=$ noise field; $\mathrm{RET}=$ retrieval; Recog $=$ recognition; $\mathrm{V}=$ verbal materials; $\mathrm{VF}=\mathrm{visual}$ fixation.

whereas MTL activations during retrieval tended to fall in more caudal regions. The strength of the observed relation between encoding/retrieval and the rostrocaudal location of the various activations was striking. For purposes of tabulation, Lepage et al. (1998) suggested an admittedly arbitrary cut-off based on xyz co-ordinates from the brain atlas of Talairach and Tournoux (1988). Activations rostral to a site defined by co-ordinates of 30 $-26-12$ (from an encoding study by $\mathrm{H}$ enke et al., 1997) were compared to activations caudal to a site defined by co-ordinates 22 $-26-8$ (from a retrieval study by Schacter et al., 1997b). U sing this cut-off, Lepage et al. (1998) noted that $87 \%$ (20/23) of the rostral sites were characterized by encoding activations, whereas $94 \%$ (29/31) of the caudal sites were characterized by retrieval activations. Perhaps even more compelling, if the encoding activations are considered separately, $91 \%$ (20/22) fall at, or anterior to, the cut-off point. If the retrieval activations are considered separately, $91 \%$ (29/32) fall at, or posterior to, the cut-off point.

Although we must refrain from placing undue emphasis on the numbers of activations that fall anterior or posterior to a dichotomous and somewhat arbitrary cut-off point- the pattern described by Lepage et al. (1998) is probably best characterized as a gradient with encoding activations ten ding to fall more rostrally, retrieval activations more caudally - the contrast to the fM RI encoding data considered earlier could hardly be more striking. We noted that too few observations are available from $\mathrm{fM}$ RI studies of retrieval to provide a meaningful picture of the location of retrieval activations, with some evidence for both anterior (Gabrieli et al., 1997) and posterior (Aguirre et al., 1996; Aguirre and D 'Esposito, 1997; Schacter et al., 1997a) activations. But the data concerning PET encoding activations are particularly interesting - and perhaps disturbing - in light of the consistent finding of posterior MTL activation during $\mathrm{fM} R \mathrm{RI}$ studies of encoding. For illustrative purposes, we applied Lepage et al.'s (1998) cut-off to fM RI encoding data with available Talairach and Tournoux (1988) co-ordinates and found that $89 \%(16 / 18)$ fall posterior to the cut-off point (see Figure 1). Such numbers must be treated with caution because relatively few $\mathrm{fM} R \mathrm{RI}$ studies have provided stereotaxic co-ordinates. H owever, in view of the fact that all fM RI encoding studies reviewed here report posterior MTL activation, we are faced with the possibility that, rather than supporting or even 
complementing each other, the fMRI and PET data contradict one another.

Alluding to a possible fM RI/PET disparity based on the findings from several studies we considered (Stern et al., 1996; Gabrieli et al., 1997; Fernandez et al., 1998), Lepage et al. (1998) raised the possibility that the observed differences between $\mathrm{fM} R \mathrm{I}$ and PET studies might reflect differences in the information provided by the two techniques. We agree that this possibility must be considered. Before accepting this conclusion, however, we wish to consider other possibilities that may account for at least some of the observed differences in the patterns of results from FM RI and PET studies.

We have examined all of the studies and data points included by Lepage et al. (1998) in their meta-analysis, relevant studies and data points that were not included, and both the explicit and implicit inclusion criteria that Lepage et al. (1998) used to generate their table. When dealing with the massive amounts of data that Lepage et al. (1998) summarized, and when making difficult decisions about inclusion/exclusion of numerous activation foci, it is virtually inevitable that oversights will occur and interpretive questions relating to inclusion/exclusion of specific points will arise. With these considerations in mind, we have generated a revised table of $M T L$ encoding and retrieval activations from PET studies (Table 2). We do not claim that this table is comprehensive; it is highly likely that we, like Lepage et al. (1998), have missed some relevant studies. The table does, however, reflect two main changes from Lepage et al.'s (1998) Table 1:

1. N ew data pointsthat meet Lepage et al.'s(1998) criteria. We have included a number of additional experimental findings that meet the inclusion criteria stated by Lepage et al. (1998). Some of the findings were available at the time Lepage et al. (1998) wrote their article and probably reflect unintentional (and, as stated above, presumably inevitable) oversights; other findings are more recent and were probably not available to Lepage et al. (1998).

2. Excluded data points. In considering Table 1 of Lepage et al. (1998), we found instances in which we believe there are grounds for excluding specific data points. These points are not incorporated in our revised table.

We now briefly consider the changes that we have made to Lepage et al.'s (1998) table on a study-by-study basis.

\section{New Data Points That Meet Lepage et al.'s (1998) Criteria}

Lepage et al. (1998) included in their table observations from studies of healthy young adults, excluding data from studies of clinical populations and older adults. They included only studies that used the PET subtraction method, which involves making pairwise comparisons between a target or experimental condition and a reference or control condition, and evaluating the differences on a voxel-by-voxel basis using a t statistic (Friston et al., 1995). Activations were included that "lie at or near the medial temporal lobes as reported by the authors of the original papers (1998, p. 315)." Lepage et al. (1998) defined an encoding condition "as yielded by any task comparison in which the target task would require elaborative processing of the materials to a larger extent than that required in the reference task (p. 315)." By contrast, they defined a retrieval condition as "one yielded by any task comparison in which the target task would involve greater use and recovery of previously experimentally encoded material than would the corresponding reference task (p. 315)." In addition, Lepage et al. (1998) "excluded all task comparisons involving the encoding of different kinds of information, as well as those involving the retrieval of different kinds of information (p. 315)." They also excluded "task comparisons in which the target task required retrieval of novel, that is previously unencoded, stimulus items (p. 315)." As best we can tell, the following observations meet these inclusion and exclusion criteria. We first consider comparisons involving encoding, and then turn to comparisons involving retrieval.

\section{Encoding Comparisons}

\section{Roskies et al. (1998)}

This study included two incidental encoding conditions, with subjects making judgments about word pairs in each condition. In the synonym condition, subjects indicated whether the two words have the same meaning; in the rhyme condition, subjects indicated whether the two words rhyme. Comparison of the synonym condition to the rhyme condition yielded an anterior M TL activation. This type of comparison between elaborative and non-elaborative encoding conditions is similar to the deep versus shallow encoding comparisons from the $\mathrm{H}$ enke et al. (1997) and Vandenberghe et al. (1996) studies included in the Lepage et al. (1998) table.

\section{Martin et al. (1995)}

Subjects were presented line drawings of common objects and performed one of three tasks: (1) naming the object, (2) generating an associated color word, or (3) generating an associated action word. Comparison of action generation to object naming did not yield MTL activation, but comparison of color generation to object naming did. As with Roskies et al. (1998), this kind of elaborative versus non-elaborative comparison is similar to those included by Lepage et al. (1998).

\section{Chao and Martin (in press)}

Following up on the findings of M artin et al. (1995), Chao and $M$ artin reported posterior MTL activation when participants generated color names compared to when they passively viewed gray-scale M ondrians.

\section{Wiggs et al. (1999)}

In a related study, W iggs et al. (1999) compared object naming and color name generation, both of which meet Lepage et al.'s (1998) encoding criteria, to a visual noise baseline. Wiggs et al. (1999) also compared color name generation to episodic retrieval of uncommon object colors. Compared to the noise baseline, 
object naming and color name generation both yielded posterior M TL activations. Compared to episodic retrieval of uncommon colors, color name generation yielded anterior M TL activation.

\section{Beauregard et al. (1998)}

In this study, incidental word encoding was compared to a lower level visual baseline. During word encoding, subjects decided whether words belonged to the category "tools." Compared to viewing a string of number signs (\#\#\#\#), word encoding resulted in left $M T L$ activation.

\section{N. Kapur et al. (1995)}

In this study, both the encoding and the retrieval of facial stimuli were compared to a rest control. From these comparisons, Lepage et al. (1998) included a retrieval MTL activation. However, a posterior MTL activation resulting from encoding (elaborating on facial stimuli by making gender classifications) was not included.

\section{Schacter et al. (1995)}

Lepage et al. (1998) included data from a retrieval comparison in which subjects made recognition judgments about a block of previously studied "impossible" objects compared to a passive viewing condition. $\mathrm{H}$ owever, they did not include analogous data showing posterior M TL activation from a retrieval comparison in which subjects made recognition judgments about a block of previously studied "possible" objects compared to a passive viewing condition. In addition, Lepage et al. (1998) did not include a comparison that meets their elaborative encoding criterion, in which subjects made possible/impossible object decisions about new (i.e., previously nonstudied) objects. For impossible objects, the object decision versus passive viewing comparison yielded a significant posterior M TL activation; there was no corresponding activation for possible objects. As with the preceding six studies, this type of elaborative decision is similar to deep versus shallow encoding comparisons that were included by Lepage et al. (1998).

\section{Bottini et al. (1994)}

Participants were presented with sentences and decided whether (1) each sentence represents a plausible metaphor, or (2) whether each sentence is plausible at the literal level. Both of these tasks require considerable elaborative processing of the sentences. In the control condition, subjects viewed a sentence-like string of eight to nine words and were asked to judge whether one of the words is an orthographically legal non-word. Compared to the control task, the combined sentence processing conditions resulted in anterior M TL activation.

\section{Price et al. (1994)}

In this study of word processing, participants performed three tasks: lexical decision on words and pseudowords, reading words aloud, and feature decision on false fonts. Across two versions of each task, the rate of stimulus presentation was varied; stimuli were presented for either 150 or 1,000 ms. At both presentation rates, compared to the baseline feature-detection task, lexical decision yielded posterior MTL activation. Lexical decision, which necessitates elaborative processing of each stimulus, is formally similar to the object decision task used in the Schacter et al. (1995) study. In addition to these foci, M T L activations related to word encoding were noted when word reading was compared to baseline. At both presentation rates, there was a more anterior MTL activation during the word reading condition. We have included these findings in our Table $2 .{ }^{2}$

\section{Bookheimer et al. (1995)}

Participants were presented words, drawings of common objects, and meaningless line drawings (similar in appearance to the false fonts of Price et al., 1994). Compared to visual scanning of the meaningless drawings, reading words aloud resulted in posterior M TL activation. Compared to the same baseline, silent object naming also yielded posterior MTL activation. Both of these comparisons are similar to the word reading versus baseline comparison of Price et al. (1994).

\section{Martin et al. (1996)}

In this study, participants passively viewed nonsense objects, named real objects from one of two categories (tools and animals), and viewed a visual noise field. As in a study by $M$ artin et al. (1997; see below for discussion) that was included by Lepage et al. (1998), compared to the noisefield baseline, passive viewing of nonsense objects yielded anterior M TL activations. This comparison also revealed a posterior M TL activation in parahippocampal gyrus. In contrast to M artin et al. (1997) and Bookheimer et al. (1995), comparison of silent object naming to the low-level baseline did not result in differential M TL activation (although, as noted by M artin et al. [1997, p. 592], this comparison did yield activity medial and superior to the hippocampus (-14 -28 4).

\section{Zelkowicz et al. (1998)}

This study included four main conditions, each of which was compared to a fixation control: (1) viewing line drawings of common objects; (2) naming drawings of common objects; (3) viewing nonsense objects; and (4) speaking (saying "H iya") while viewing nonsense objects. Asin the studies by M artin et al. (1996,

${ }^{2}$ We thank M. Lepage for calling our attention to the Price et al. (1994) study. N ote that, although the lexical decision condition in Price et al. (1994) clearly satisfies Lepage et al.'s (1998) encoding criteria, because Lepage et al. (1998) emphasized that encoding conditions involve either elaborative processing or intentional learning (or both), simple word reading may seem to violate these criteria. However, it is possible that word reading involves greater levels of encoding than does the control condition, and therefore inclusion of this comparison may be warranted (M. Lepage, personal communication). Similar considerations apply to the studies by Bookheimer et al. (1995), Martin et al. (1996), and Zelkowicz et al. (1998), as well as a study by Martin et al. (1997) that was originally included by Lepage et al. (1998). 
1997), viewing nonsense objects compared to the lower level baseline yielded an anterior M TL activation.

\section{Taylor et al. (1997)}

When visually presented with a word, people automatically read it. As discussed when considering the word reading results of Price et al. (1994) and Bookheimer et al. (1995), word reading likely results in greater stimulus encoding compared to lower level baselines. Taylor et al. (1997) examined automatic word reading using the well-known Stroop effect, which occurs when automatic reading of a word interferes with naming the color in which the word is printed. In Taylor et al.'s paradigm, subjects named the color in which various stimuli (e.g., words and symbols) were presented. A condition that requires naming the color of a word should entail greater word reading and encoding than a condition that requires naming the color in which meaningless symbols (\#\#\#) are printed. Taylor et al. (1997) reported greater posterior M TL activity during color naming of taboo words (which elicits automatic word reading), compared to color naming of symbols (which does not elicit automatic word reading).

\section{Kopelman et al. (1998)}

In a novel encoding condition, participants intentionally memorized three lists of 15 words they had not previously seen. Compared to a control condition in which each list consisted of a single word repeated 15 times, novel encoding yielded a posterior MTL activation. When the novel encoding condition was combined with one in which participants memorized a previously exposed list (repeated encoding), comparison to the single word control condition revealed a more anterior MTL activation. Kopelman et al. (1998) performed additional correlational analyses to assess regions demonstrating an association between incremental learning (i.e., a difference score reflecting gains in learning across trials) and blood flow. When all encoding trials were included, a posterior MTL region was associated with incremental learning (-26 -30 -16; not included in Table 2 because such analyses are excluded by Lepage et al.'s criteria). But when only the repeated encoding trials were included, a more anterior M TL region was associated with incremental learning (-28 -22 0; also not included in Table 2).

\section{Decety et al. (1997)}

In this study, subjects were instructed to learn meaningful and meaningless actions in order to be able either to recognize or imitate them subsequently. Lepage et al. (1998) included a meaningful versus meaningless comparison that collapsed across the recognition and imitation conditions, yielding an anterior M TL activation (24 0 -24) and a slightly more posterior one (-40 -22 -12). However, when the meaningful versus meaningless comparison was performed separately for the recognition and imitation conditions, it became clear that there was an anterior activation for the imitate condition and a posterior activation for the recognition condition. Because the combined comparison serves to mask these two distinct foci, we have included in Table 2 the more specific comparisons that reflect the separate contributions of the recognition and imitation conditions. We have left in Table 2 one point from the more general comparison (-40 -22 -12) that appears to be distinct from either of the foci yielded by the two specific comparisons. We excluded the other point from the more general comparison (24 0 -20) because it is nearly identical to one of the foci from the specific comparisons (26 0 $-20)$.

\section{Maguire et al. (1996)}

The encoding task required subjects to view a film depicting navigation through a town; they were instructed to try to memorize a route so that they could later find their way. Compared to control conditions in which participants passively watched non-navigation films, Maguire et al. (1996) reported bilateral activation of the hippocampal formation. ${ }^{3}$

\section{Maguire et al. (1998b)}

In the encoding condition, participants explored and memorized an environment containing objects and textures. Compared to a motion detection control condition that included similar textures, encoding yielded significant activation in the parahippocampal cortex.

Both M aguire et al. (1996) and M aguire et al. (1998b) appear to meet Lepage et al.'s (1998, p.315) encoding criterion of "any task comparison in which the target task would require elaborative processing of the materials to a larger extent than that required in the reference task." Because the reference conditions did not contain precisely the same materials as the encoding conditions, these comparisons might involve "different kinds of information" that are excluded by Lepage et al. (1998). H owever, the same can be said of numerous points that were included by Lepage et al. (1998), mainly those involving comparisons to low level baselines, fixation, or rest. For example, all of the included points from M artin et al. (1997), which account for 8 of the 20 anterior M TL encoding activations in Lepage et al.'s table, are based on comparisons between encoding of various stimulus types (words, nonsense words, objects, or nonsense objects) and a baseline that involved viewing of visual noise patterns. W hile it is clear that the target condition involves encoding to a greater extent than the reference condition, the target condition also involves "different kinds of information" than the reference condition; differences

${ }^{3}$ We thank E. Maguire for providing stereotaxic co-ordinates. Maguire et al. (1996) also compared the navigation encoding condition to a condition requiring intentional learning of a non-navigational film. Comparison of navigation to non-navigation encoding, which is a comparison across kinds of information and therefore violates Lepage et al.'s (1998) criteria, yielded anterior (34 -18 -16) and posterior (22 -36 -12; -16 -42 -8) MTL activation. 
between the target and reference conditions could thus be attributable to encoding, to different kinds of information, or both. Even in the aforementioned study by D ecety et al. (1997), which used a somewhat tighter comparison, the reference condition (studying meaningless actions) did not contain the same information as did the encoding condition (studying meaningful actions). Thus, our best reading of Lepage et al.'s (1998) criteria indicates that the findings of M aguire et al. $(1996,1998 b)$ merit inclusion in Table 2.

\section{Retrieval Comparisons}

\section{Maguire et al. (1998a)}

Just prior to scanning, subjects explored a "virtual town." In two subsequent scans, they were instructed to find their way to a location in the town, thus requiring retrieval of an internal representation of the environment. Compared to a control condition that involved passive navigation, retrieval scans in which subjects succesfully reached the goal location yielded activation of the right hippocampus. C omparison of these successful retrieval scans to scans in which participants failed to reach the goal location resulted in bilateral hippocampal activation.

\section{Schacter et al. (1997b)}

Subjects made old/new recognition judgments about previously studied possible objects that were presented in the same size and orientation in which they were studied or in a different size or orientation. Baseline conditions consisted of passive object viewing and old/new judgmentsabout blocks of new objects. Comparison of the same object condition to either of these baselines yielded multiple M TL activations that were included in Lepage et al.'s (1998) table. H owever, a similar comparison of orientation change to the new object baseline that also yielded MTL activation was not included.

\section{Petersson et al. (1997)}

In the baseline condition, subjects mentally "filled in" or completed the contours of abstract designs. In the "novel" retrieval condition, subjects recalled abstract designs from a previously studied list. In the "trained" retrieval condition, subjects recalled the same designs that they had recalled on several previous occasions. Subtraction of the trained from the novel retrieval condition revealed multiple M TL activations. Because these results could be produced either by retrieval processes or stimulus novelty, they are excluded by Lepage et al.'s (1998) criteria and we do not include them in our Table 2. H owever, Petersson et al. (1997) also examined M TL activation by comparing the novel retrieval condition with the baseline condition (using the regions identified in the novel versus trained recall comparison as a mask). The retrieval minus baseline subtraction yielded an anterior M TL activation. This comparison is not plagued by the confounding with stimulus novelty that characterizes the novel versus trained subtraction, and thus seems appropriate for inclusion in Table 2 (in addition, a hypothesis- driven region-of-interest analysis revealed right hippocampal (31 $-29-8)$ and bilateral parahippocampal (29-23 -20; -29 -23 -20) effects; we do not include these points in Table 2 because they do not involve voxel-by-voxel comparisons required by Lepage et al.).

\section{Heckers et al. (1998)}

This experiment examined retrieval processes in schizophrenic patients and control subjects with a high/low recall procedure developed in an earlier study (Schacter et al., 1996a) that was included in Lepage et al.'s (1998) table. Though the schizophrenic patients are excluded by Lepage et al.'s (1998) criteria, we include hippocampal and parahippocampal activations from the normal control group.

\section{Excluded Data Points}

\section{Grasby et al. (1993)}

Lepage et al. (1998) included two points from this study as retrieval activations. However, scanning was performed during conditions in which subjects both encoded and retrieved word lists. It is thus not possible to determine whether these activations result from encoding or retrieval because, as noted subsequently by Grasby and colleagues (Kopelman et al., 1998, p.884), the two are confounded in this study.

\section{Martin et al. (1997)}

Among the eight activations from this study included by Lepage et al. (1998) were comparisons involving (1) word encoding minus viewing of noise patterns, (2) picture encoding minus viewing of noise patterns, and (3) word + picture encoding minus viewing of noise patterns. The two MTL activations yielded by (3) $(-34-16-20 ; 22-18-20)$ are quite similar to the foci in (1) (-33-15-20) and (2) (24 -24-20), respectively, and thus would appear redundant. Accordingly, we have excluded the two activations in the combined comparison (3).

Finally, we also note interpretive complications that arise from a study by Roland and G ulyás (1995). Lepage et al. (1998) included four M TL activations from this study, two in which an encoding condition was compared to a rest baseline, and two in which a retrieval condition (recognition memory) was compared to a rest baseline. For the encoding foci, Roland and Gulyás (1995) also reported a direct comparison of the encoding and the retrieval conditions that yielded no evidence of $M T L$ activation. This finding implies that the MTL activations from the encoding versus rest comparison are not specifically produced by encoding processes but are instead attributable to other differences (e.g., stimulus properties, task difficulty) between the encoding and rest conditions. We have left the Roland and Gulyás (1995) points in Table 2 because focused comparisons of the kind they reported are not available for the other studies in the table that involve comparisons to rest or fixation. N onetheless, as in our earlier discussion of a hippocampal activation in the Wagner et al. (1998) fM RI study, we think it is important to underscore the interpretive ambiguities that arise when attempting to 
make inferences about encoding or retrieval based on relatively unconstrained comparisons to rest or fixation conditions.

\section{SUMMARY AND FURTHER CONSIDERATIONS}

$\mathrm{H}$ aving discussed findings that should be included or excluded from the encoding/retrieval meta-analysis based on Lepage et al.'s (1998) criteria, we can now consider some implications for the rostrocaudal distribution of encoding and retrieval activations. Even with our deletions and additions, the general pattern described by Lepage et al. (1998) is still apparent in Table 2, with encoding activations tending to occupy anterior sites and retrieval activations tending to occupy more posterior sties. However, the rostrocaudal gradient of encoding and retrieval activations is now less sharply defined than in Lepage et al. (1998). Encoding activations are observed frequently in both anterior and posterior sites, with a weak tendency for more anterior than posterior activations; retrieval activations show a more marked tendency to occupy more posterior than anterior sites. To provide some quantitative grounding for these observations, recall that $91 \%$ (20/22) of encoding activations in Lepage et al. (1998) fell at or anterior to their cut-off point, and $91 \%$ (29/32) of retrieval activations fell at or posterior to the cut-off point. Our revised tabulation (we do not include in this tabulation the seven points that fall directly on the Lepage et al. cut-off of $y=-26$ ) indicates that $58 \%(26 / 45)$ of encoding activations fall anterior to the Lepage et al. (1998) cut-off, whereas $80 \%$ (28/35) of retrieval activations fall posterior to this cut-off (for a schematic depiction, see Figure 2).

Relating these findings to our earlier discussion of $\mathrm{fMRI}$ studies, we noted previously that because encoding activations were associated almost exclusively with anterior MTL sites in Lepage et al.'s (1998) analysis, PET encoding data seemed to contradict the finding of posterior M TL activation reported in all of the fM RI studies we considered. H owever, the observation that only $58 \%$ of PET encoding activations occupy anterior sites indicates that the contradiction between PET and fM RI data was more apparent than real. Consistent with this point, because a number of studies contribute multiple points to Table 2 , it should be emphasized that fourteen studies contributed encoding activations anterior to the cut-off, whereas fifteen studies contributed encoding activations posterior to the cut-off. It therefore seems safe to conclude that even with our adherence to Lepage et al.'s (1998) highly restrictive criteria, PET encoding activations occur frequently in posterior MTL regions, thereby removing the contradiction with $\mathrm{FMRI}$ data. But it seems equally clear that while both $\mathrm{FM} \mathrm{RI}$ and PET have provided evidence of encodingrelated activations in the posterior MTL, only PET has consistently provided evidence of more anterior MTL activations. We discuss possible reasons for this state of affairs shortly.

\section{ADDITIONAL DATA POINTS THAT DO NOT MEET LEPAGE ET AL.'S (1998) CRITERIA}

We next briefly mention several activations from studies that do not meet all of the Lepage et al.'s (1998) criteria.

As noted earlier, Lepage et al. (1998) restricted their focus to studies using the subtraction method. Nyberg et al. (1996) reported significant correlations between MTL activity and successful retrieval (recognition) of previously studied words. In an initial experiment, they reported $a+.82$ correlation between recognition performance and blood flow at a voxel in the left anterior MTL (near the amygdala; -24 2 -16). In a second experiment, they reported a similar correlation at a voxel near the left hippocampus (-24 -24 -12). Alkire et al. (1998) used a different type of correlational analysis to examine M TL activity during word encoding. Alkire et al. (1998) scanned subjects while they listened to a repeated sequence of unrelated words. Twentyfour hours later (outside the scanner), subjects attempted to recall the previously studied words. Alkire et al. (1998) reported a strong positive correlation $(+.91)$ between activity in a left posterior MTL region (-25 - $-40-4)$ during the encoding phase of the experiment and the number of words recalled on the subsequent memory test. ${ }^{4}$ These results converge with the previously mentioned event-related fMRI findings of Wagner et al. (1998) indicating that left posterior M TL activity during word encoding is associated with subsequent remembering and forgetting on a recognition test.

Finally, we note a recent study by M ontaldi et al. (1998) using single photon emission computed tomography (SPECT) to examine associative encoding of novel complex scenes. During scanning, participants memorized each of 60 pictures for a later recognition task in one condition. In another condition, participants performed a perceptual matching task in which a target scene was presented simultaneously with two choice scenes, and they had to indicate which of the two choice scenes matched the target (see Grady et al., 1995). When comparing the encoding condition to the matching condition, Montaldi et al. (1998) found significant activation in left MTL (-16 -10 -16; -24 -14 -24). We did not include these findings in Table 2, which was restricted to PET studies, nor have we systematically examined other SPECT studies. But Montaldi et al.'s (1998) data are consistent with the pattern described by Lepage et al. (1998) and should be noted.

\section{CONCLUSIONS}

We began by summarizing $f M R I$ studies of episodic encoding and retrieval that have obtained evidence of MTL activation.

${ }^{4}$ We thank M. Alkire and L. Cahill for providing stereotaxic co-ordinates. 
Although only a few studies have examined retrieval processes, studies concerned with encoding processes have consistently obtained evidence of posterior MTL activation across various materials, conditions, and types of analyses. Noting the recent meta-analysis of PET studies by Lepage et al. (1998) that supported precisely theopposite conclusion - that episodic encoding is strongly, almost exclusively, associated with anterior MTL activation - we raised the possibility that $\mathrm{FMRI}$ and PET data provide contradictory evidence. After considering the criteria and evidence used by Lepage et al. (1998) in their meta-analysis, we observed that the association between episodic encoding and anterior MTL activation may not be as strong as it appeared initially. When we included and excluded studies based on Lepage et al.'s (1998) criteria, we found that encoding activations in PET studies are observed frequently in both anterior and posterior M T L sites, with a less clearly defined tendency for the rostrocaudal gradient than was initially reported by Lepage et al. (1998).

These observations indicate that results from $\mathrm{FM} \mathrm{RI}$ and PET studies do not lead to contradictory conclusions regarding the rostrocaudal locus of MTL activations during episodic encoding. N onetheless, as noted earlier it seems clear that PET studies of encoding more frequently report anterior $M T L$ activations than do $\mathrm{fM} \mathrm{RI} \mathrm{studies} \mathrm{of} \mathrm{encoding.} \mathrm{O} \mathrm{ne} \mathrm{possibility} \mathrm{is} \mathrm{that} \mathrm{PET} \mathrm{is} \mathrm{more}$ sensitive than $\mathrm{FMRI}$ to changes in the anterior MTL. FMRI is known to be characterized by susceptibility artifacts that can be pronounced in anterior M T L regions (e.g., O jemann et al., 1997). Such artifacts may make it difficult to detect anterior MTL activity that is revealed by PET, whereas both techniques are sensitive to activations in posterior M TL. In their fM RI study of environmental encoding, Aguirre et al. (1996, p.825) considered the effects of susceptibility artifact on anterior M TL signal. They noted that although "the $\mathrm{fMRI}$ signal was lost from the most anterior and inferior portion of the temporal lobes," "the hippocampal formation, over its entire extent, was imaged in all subjects." Inspection of data from our own laboratory has also revealed evidence of susceptibility artifact in the most anterior regions of the $M T L$ (i.e., temporal pole), but not in the hippocampal formation (Figure 3; note, however, that the signalto-noise ratio is higher in posterior $M T L$ than in anterior $M T L$ ). Similarly, data from O jemann et al. (1997, figure 1) indicate that susceptibility to signal loss in M TL is restricted to regions near the temporal pole (note that susceptibility artifacts are also found in more lateral temporal regions).

Consistent with these observations, several of the studies considered earlier have provided some evidence that fM RI can detect more anterior M TL signal changes. Gabrieli et al. (1997) reported anterior MTL (subiculum) activation during memory retrieval, Rombouts et al. (1997) reported some evidence of anterior MTL (hippocampal) activation during encoding in addition to posterior M TL activation, and Wagner et al. (1998) reported an anterior MTL (hippocampal) activation as well as posterior MTL (parahippocampal) activation when comparing word encoding to visual fixation in a blocked design experiment (as stated previously, the hippocampal activation must be treated cautiously). Though not conclusive, the foregoing considerations suggest that failures to detect anterior MTL activations during encoding in fMRI studies are probably not attributable to susceptibility artifact.

In contrast to the idea that rostrocaudal differences in MTL encoding activations are attributable to differences between fM RI and PET, we suggest an alternative possibility. Different paradigms have often been used in $\mathrm{FM} \mathrm{RI}$ and PET studies of encoding, thus complicating direct comparison between the two imaging modalities. More specifically, considering the fourteen PET studies that have produced evidence for anterior M TL encoding activations, we note that the experimental paradigms used by Decety et al. (1997; this study also yielded a posterior M TL encoding activation), Dolan and Fletcher (1997), Henke et al. (1997), Roland and Gulyás (1995; this study also yielded a posterior M TL encoding activation), Roskies et al. (1998), and Wiggs et al. (1999; this study also yielded posterior MTL activations) have not been examined in fM RI studies, nor have the nonsense object encoding conditions that yielded anterior M TL activation in M artin et al. $(1996,1997)$ and Zelkowicz et al. (1998). Kopelman et al. (1998) reported anterior M T L activation when comparing novel and repeated word encoding conditions to a control; no fM RI studies have reported analogous comparisons. Interestingly, Kopelman et al.'s separate comparison of novel word encoding to the control condition, which is similar to that described by Fernandez et al. (1998) and Kelley et al. (1998) in fM RI studies, yielded posterior M TL activation.

Experimental conditions roughly similar to the word and object encoding conditions from M artin et al. (1997), Wiggs et al. (1999), and Price et al. (1994) have been used in fM RI studies (Kelley et al., 1998; Wagner et al., 1998). It is not clear why these studies have yielded different outcomes, but it may be worth noting that there are inconsistencies among PET studies as well as between PET and fM RI studies. In contrast to the anterior M TL activation observed by Price et al. (1994) during word reading and Martin et al. (1997) during object naming, Bookheimer et al. (1995) reported posterior M TL activation during word reading and object naming. Similarly, Wiggs et al. (1999) reported posterior M TL activation when comparing object naming to the same visual noise baseline used by Martin et al. (1997). Two further PET studies that have produced anterior M TL activation used procedures that are similar to those used in $\mathrm{fM}$ RI studies. Vandenberghe et al. (1997; PET) compared deep and shallow verbal encoding, as did the study by Wagner et al. (1998; fM RI) that produced posterior M T L activation. H owever, there are some procedural differences between the two studies, most notably that Vandenberghe et al. used word triplets and Wagner et al. used single words. $\mathrm{H}$ axby et al. (1996; PET ) compared intentional face encoding to a face matching control. The anterior M T L activation in this study differs from the posterior activation found by Kelley et al. (1998; fM RI) when comparing intentional face encoding to passive face viewing. We also note, however, that $\mathrm{N}$. K apur et al. 


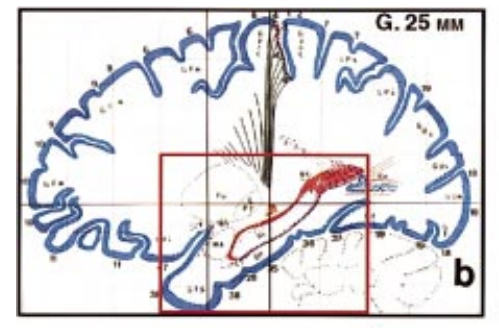

\section{MTL ACTIVATIONS DURING ENCODING AND RETRIEVAL: FMRI STUDIES}
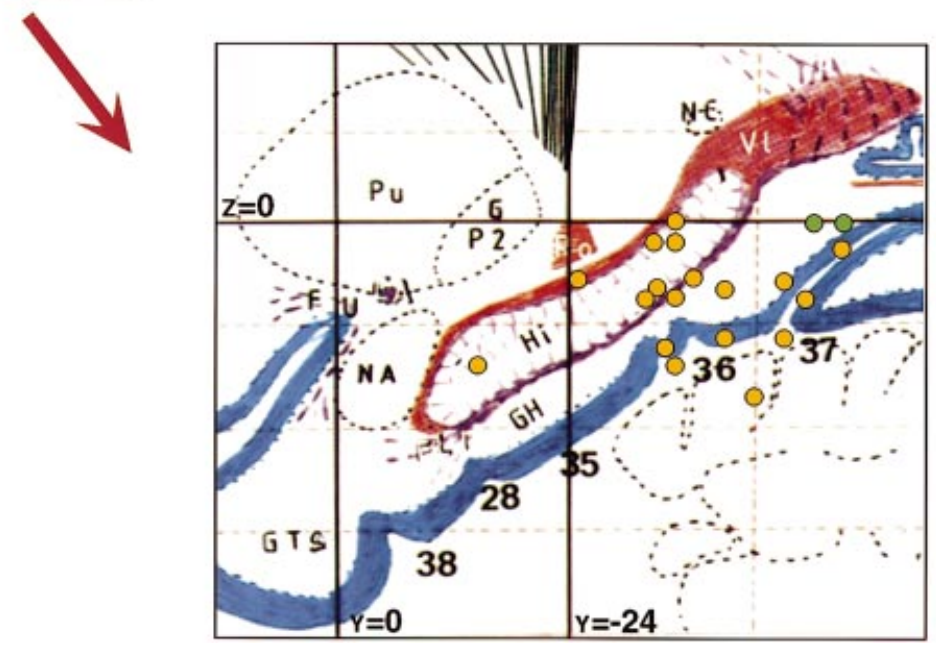

FIGURE 1. Schematic rendering of $F M R I$ encoding and re trieval activations reported by authors of the original studies to fall in the medial temporal lobe. Foci from both the left and right hemispheres are shown on a single sagittal plane taken from the Talairach and Tournoux (1988) atlas (25 $\mathrm{mm}$ lateral to the midline). 0 verlapping activations were offset slightly. Activation foci shown in the figure derive from studies that reported stereotaxic co-ordinates; additional activations from studies that did not report stereotaxic co-ordinates are summarized in Table 1.

(1995; PET) reported posterior activation during incidental encoding of faces compared to a resting baseline.

$O$ verall, then, it is clear that few of the PET studies that produced anterior MTL activations during encoding have used paradigms or comparisons that are similar to those that have been used in fM RI encoding studies. When similar procedures have been used, in a number of cases similar results have been obtained. Consistent with this observation, we note converging posterior M TL activations in the closely comparable environmental encoding studies of M aguire et al. (1996, 1998b; PET) and Aguirre and colleagues (Aguirre et al., 1996; Aguirre and D'Esposito, 1997; fMRI).

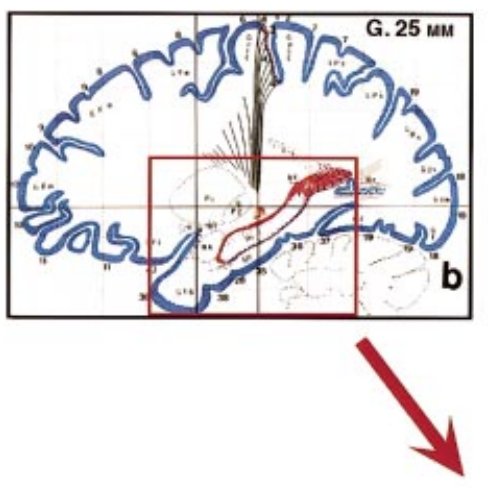

\section{MTL ACTIVATIONS DURING ENCODING AND RETRIEVAL: PET STUDIES}

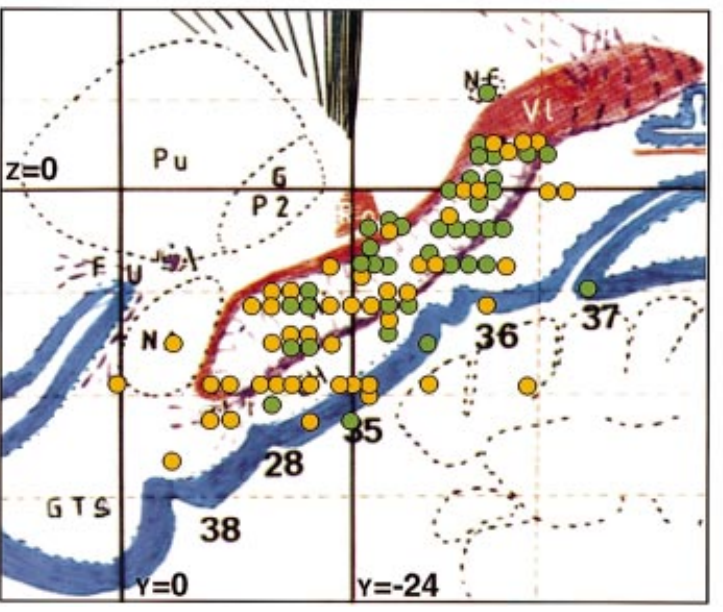

FIGURE 2. Schematic rendering of PET encoding and retrieval activations reported by authors of the original studies to fall in the medial temporal lobe that meet the inclusion criteria of Lepage et al. (1998). Foci from both the left and right hemispheres are shown on a single sagittal plane taken from the Talairach and Tournoux (1988) atlas $(25 \mathrm{~mm}$ lateral to the midline). O verlapping activations were offset slightly. 


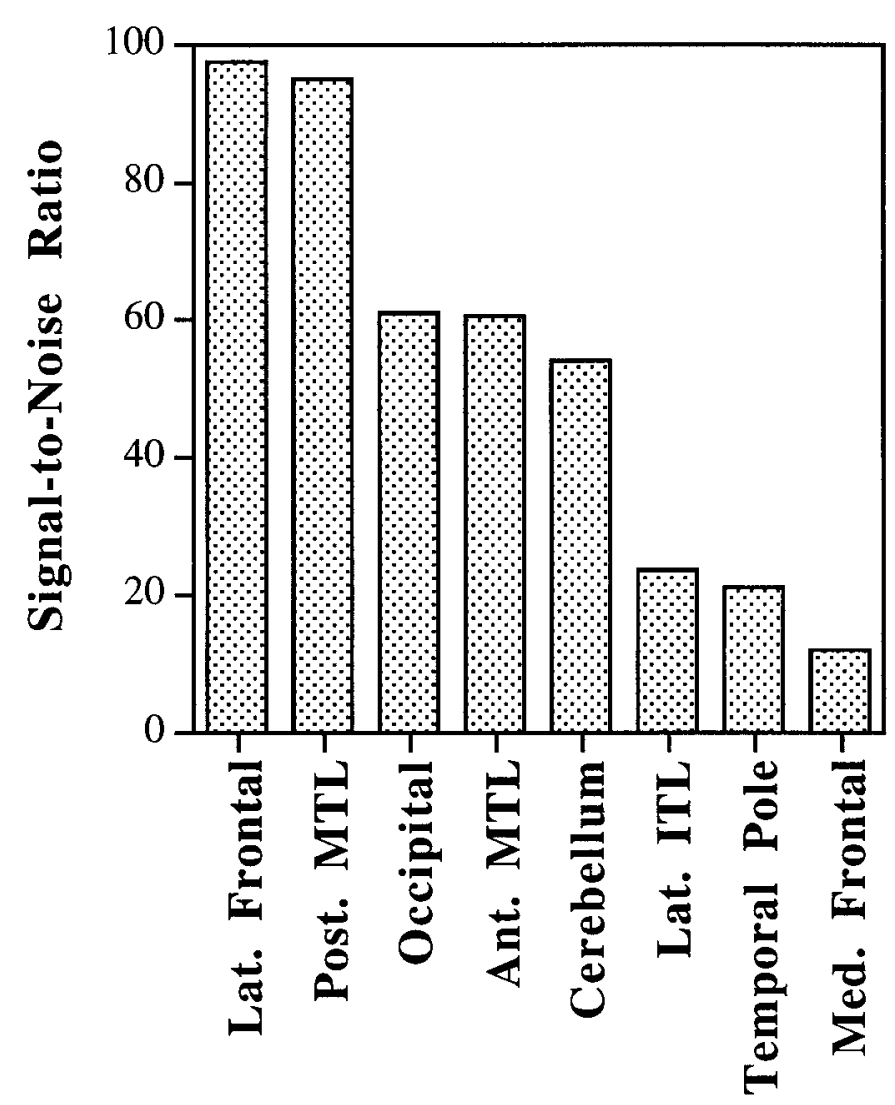

FIGURE 3. Mean signal-to-noise ratios (SNR) for selected neural regions. D ata were acquired while five subjects visually fixated for $100 \mathrm{sec}$ using a 3.0T GE Signa scanner (gradient-echo EPI sequence, TR $=2 \mathrm{sec}, \mathrm{TE}=\mathbf{4 0} \mathrm{msec}, 16$ slices parallel to anterior-commissure/ posterior-commissure plane). Although SNR is higher in posterior than anterior medial-temporal lobe (MTL), both anterior and posterior MTL regions have SNRs that are comparable to regions typically not associated with susceptibility artifact, including occipital cortex and cerebellum. Regions adjacent to bone and air sinuses, such as lateral inferior temporal (ITL), medial inferior frontal, and inferior temporal pole areas, demonstrate considerable susceptibilityinduced signal loss. Lat. = lateral; M ed. = medial.

Based on these considerations, we emphasize two points. First, both anterior and posterior regions of the MTL are involved in memory encoding. Second, rather than attributing the relative absence of anterior MTL activations in $\mathrm{FM} \mathrm{RI}$ encoding studies to fundamental differences between PET and $\mathrm{FMRI}$, we suggest thatsome of those differences reflect differences in the behavioral procedures that have been used in PET and FM RI studies. From this perspective, we expect that future PET and $F M R I$ encoding studies using closely comparable behavioral procedures will yield convergent results.

Future studies may also help to clarify a related question that emerges from our analysis: what are the distinguishing characteristics of studies that produced anterior M TL encoding activations compared to those that produced more posterior activations? In considering the fourteen PET encoding studies that reported clear evidence of anterior M TL activations, it is difficult to discern any critical feature or features that set them apart from studies that produced posterior MTL encoding activations. Both sets of studies include various material types (words, faces, objects, and scenes), intentional and incidental encoding instructions, comparisons to various kinds of control conditions, and so forth. Three of the studies yielding anterior M TL activations contained withinexperiment manipulations that also produced more posterior MTL activations (Price, et al., 1994; Decety et al., 1997; Kopelman, et al., 1998; see earlier descriptions), but the results of these studies do not converge in a clear manner.

We do note, however, that five of the PET studies that yielded anterior M TL encoding activations specifically required subjects to focus on the relations among multiple stimuli - either word pairs (Dolan and Fletcher, 1997; Roskies et al., 1998), word triplets (Vandenberghe et al., 1996), pictures of people and houses (H enke et al., 1997), or sentences (Bottini et al., 1994). O nly one of the PET studies that yielded posterior M TL encoding activations required processing of multiple stimuli (word pairs; K apur et al., 1996). In addition, learning of complex environments (associated with posterior M TL activations in M aguire, 1996, 1998b, and also in Aguirre et al., 1996) might involve relational processes. Interestingly, one of the few fM RI studies to report evidence of more anterior MTL encoding activation (Rombouts et al., 1997)required relational processing of multiple stimuli. Clearly, a requirement for relational processing cannot explain all instances of anterior MTL encoding activations. Nonetheless, given the emphasis on relational processing in recent accounts of hippocampal function (compare Cohen and Eichenbaum, 1993; Rudy and Sutherland, 1994; Squire, 1994; Eichenbaum and Bunsey, 1995), future studies should systematically test the hypothesis that relational encoding, in comparison to non-relational encoding, tends to be preferentially associated with anterior MTL activations. Such studies could contribute to a more general attempt to determine what (if any) aspects of encoding tasks, experimental materials, or other relevant features tend to be associated with anterior versus posterior M TL encoding activations.

We have focused on results from encoding conditions because of the impressive convergence of evidence from $\mathrm{fM} \mathrm{RI}$ experiments concerning the role of posterior M TL in encoding processes, and the apparent contradiction indicated by Lepage et al.'s (1998) summary of PET studies. But we should not lose sight of the fact that even after our modifications to Lepage et al.'s (1998) table, the PET evidence still shows a tendency, though less dramatic than initially described, for retrieval activations to be located in posterior MTL regions. Further evidence from PET and FM RI studies is required to determine definitively whether retrieval activations are preferentially associated with posterior as compared to anterior M T L regions.

If this pattern holds, then it will become extremely important to determine why episodic encoding involves both anterior and posterior M T L - as current PET and FM RI data clearly indicatewhereas episodic retrieval is associated mainly with posterior M TL. O ne intriguing possibility concerns the relational processing hypothesis we suggested as an account of at least some anterior M TL encoding activations. We are unaware of any PET or fM RI studies of retrieval that have specifically required relational processing of multiple stimuli in a recall or recognition test. If 
relational processing tends to produce anterior $M T L$ activations during encoding, it may also produce similar anterior MTL activations during retrieval. Consistent with this possibility, 0 wen et al. (1996) reported anterior MTL activation (28 -13 -29) during a retrieval task that required participants to remember the relation between objects and their locations, compared to a task that required memory for location only. Future studies could evaluate the hypothesis by explicitly comparing relational and non-relational retrieval tasks (e.g., Eichenbaum and Bunsey, 1995). Furthermore, direct comparisons between encoding and retrieval tasks - both relational and non-relational - could help to determine whether variations in rostrocaudal location of $\mathrm{MTL}$ activations are associated with differences between encoding and retrieval per se, or to differences that cut across encoding and retrieval, such as relational versus non-relational processing.

Although we have raised questions about some of the conclusions to emerge from Lepage et al.'s (1998) meta-analysis, we believe that they have performed an important task by organizing a large body of findings and bringing into sharp focus questions concerning the rostrocaudal distribution of MTL activation during episodic encoding and retrieval. Clarifying and understanding the issues raised by their analysis and by ours will no doubt help to achieve the goal of using neuroimaging techniques to elucidate the role of specific M TL regions in encoding and retrieval processes.

\section{Acknowledgments}

We thank R. Buckner, T. Curran, J. G abrieli, W. Koutstaal, M. Lepage, and E. Tulving for helpful comments and suggestions, and $\mathrm{C}$. Racine for help with preparation of the manuscript.

\section{REFERENCES}

Aguirre GK, D'Esposito M. 1997. Environmental knowledge is subserved by separable dorsal/ventral neural areas. J N eurosci 17:25122518.

Aguirre GK, D etre J, Alsop D, D 'Esposito M . 1996. The parahippocampus subserves topographical learning in man. Cereb Cortex 6:823829.

Aguirre GK, Zarahn E, D 'Esposito M . 1997 A test of the relationship between hippocampal activity and correct word recognition with trial-based fM RI. C ognit N eurosci Soc 4th Annu M eet 4:63.

Aguirre GK, Zarahn E, D'Esposito M. 1998. An area within human ventral cortex sensitive to "building" stimuli: Evidence and implications. Neuron 21:373-383.

Alkire M T, H aier R, Fallon JH , Cahill, L. 1998. Hippocampal, but not amygdala, activity at encoding correlates with long-term, free recall of non-emotional information. Proc N atl Acad Sci 95:14506-14510.

Beauregard M, Gold D, Evans PC, Chertkow H. 1998. A role for the hippocampal formation in implicit memory: a 3-D PET study. N euroReport 9:1867-1873.

Blaxton TA, Bookheimer SY, Zeffiro TA, Figlozzi CM, Gaillard WD, Theodore WH .1996. Functional mapping of human memory using PET: comparisons of conceptual and perceptual tasks. Can J Exp Psychol 50:42-56.

Bookheimer SY, Z effiro TA, Blaxton T, Gaillard W, Theodore W. 1995.
Regional cerebral blood flow during object naming and word reading. H um Brain M ap 3:93-106.

Bottini G, Corcoran R., Sterzi R, Paulesu E, Schenone P, Scarpa P, Frackowiak RSJ, Frith CD. 1994. The role of the right hemisphere in the interpretation of figurate aspects of language: A positron emission tomography activation study. Brain 117:1241-1253.

Brewer J B, Zhao Z, G lover G H, G abrieli JDE. 1998. M aking memories: brain activity that predicts whether visual experiences will be remembered or forgotten. Science 281:1185-1187.

Buckner RL, Koutstaal W. 1998. Functional neuroimaging studies of encoding, priming, and explicit memory retrieval. Proc $\mathrm{N}$ atl Acad Sci USA 95:891-898.

Buckner RL, Petersen SE, O jemann JG, M iezin FM, Squire LR, Raichle ME. 1995. Functional anatomical studies of explicit and implicit memory retrieval tasks. J N eurosci 15:12-29.

Buckner RL, Bandettini P, O 'C raven K, Savoy R, Petersen SE, Raichle M E, Rosen BR.1996. D etection of cortical activation during averaged single trials of a cognitive task using functional magnetic resonance imaging. Proc N atl Acad Sci 93:14878-14883.

Cabeza R, N yberg L.1997. Imaging cognition: An empirical review of PET studies with normal subjects. J Cognit N eurosci 9:1-26.

Chao LL, M artin A. C ortical regions associated with perceiving, naming, and knowing about colors. J Cognit N eurosci (in press).

Cohen N J, Eichenbaum H . 1993. M emory, amnesia, and the hippocampal system. Cambridge M A: M IT Press.

Craik FIM , Lockhart RS.1972. Levels of processing: A framework for memory research. J Verb Learn Verb Behav 11:671-684.

Dale A, Buckner R. 1997. Selective averaging of rapidly presented individual trials using fM RI. H um Brain M ap 5:329-340.

D ecety J, Grezes J, C ostes N, Perani D , Jeannerod M , Procyk E, Grassi F, Fazio F. 1997. Brain activity during observation of actions: Influence of action content and subject's strategy. Brain 120:1763-1777.

D emb JB, D esmond JE, Wagner AD, Vaidya CJ, Glouer GH, Gabrieli JDE. 1995. Semantic encoding and retrieval in the left inferior prefrontal cortex: A functional M RI study of task difficulty and process specificity. J N eurosci 15:5870-5878.

Dolan R, Fletcher P. 1997. Dissociating prefrontal and hippocampal function in episodic memory encoding. $N$ ature 388:582-585.

Eichenbaum H, Bunsey M. 1995. On the binding of associations in memory: Clues from studies on the role of the hippocampal region in paired-associate memory. Curr D irect Psychol Sci 4:19-23.

Elliot R, D olan R. 1998. N eural response during preference and memory judgments for subliminally presented stimuli: a functional neuroimaging study. J N eurosci 18:4697-4704.

Epstein R, Kanwisher N. 1998. A cortical representation of the local visual environment. N ature 397:598-601.

Fernandez G, Weyerts H, Schrader-Bolsche M, Tendolkar I, Smid H G O M , Tempelmann C, H inrichs $H$, Scheich $H$, Elger CE, M angun GR, H einze H-J. 1998. Succesfful verbal encoding into episodic memory engages the posterior hippocampus: a parametrically anaIyzed functional magnetic resonancestudy. J N eurosci 18:1841-1847.

Fletcher PC, Frith CD, Grasby PM, Shallice T, Frackowiak RSJ, D olan RJ. 1995. Brain systems for encoding and retrieval of auditory verbal memory: An in vivo study in humans. Brain 118:401-416.

Friston, KJ, Holmes AP, Worsley KJ, Poline J-P, Frith CD, Frackowiak RS]. 1995. Statistical parametric maps in functional imaging: $A$ general linear approach. H um Brain M ap 2:189-210.

Fujii T, O kuda J, Kawashima R, Yamadori A, Fukatsu R, Suzuki K, Ito M, Goto R, Fukuda H. 1997. Different roles of the left and right parahippocampal regions in verbal recognition: A PET study. N euroReport 8:1113-1117.

Gabrieli JDE, Brewer JB, Desmond JE, Glover GH. 1997. Separate neural bases of two fundamental memory processes in the human medial temporal lobe. Science 276:264-266.

Gardiner JM , Java RI. 1993. Recognising and remembering. In: Collins 
AF, Gathercole SE, Conway MA, Morris PE, eds. Theories of memory. H ove, United Kingdom: Erlbaum. p 163-188.

G haem $O$, M ellet $E$, Crivello F, Tzourio N, M azoyer B, Berthoz A, D enis $M$. 1997. M ental navigation along memorized routes activates the hippocampus, precuneus, and insula. N euroReport 8:739-744.

Grady $C L$, M clntosh $A R$, Horwitz $B, M$ aisog JM, Ungerleider $L G$, Mentis MJ, Pietrini P, Schapiro M B, H axby JV. 1995. Age-related reductions in human recognition memory due to impaired encoding. Science 269:218-221.

Grasby PM, Frith CD, Friston KJ, Bench C, Frackowiak RSJ, Dolan RJ.1993. Functional mapping of brain areas implicated in auditoryverbal memory function. Brain 166:1-20.

$\mathrm{H}$ axby JV. 1996. M edial temporal lobe imaging. $\mathrm{N}$ ature 380:669-670.

H axby JV, Ungerleider L, H orwitz B, M aisog J, Rappaport S, Grady C. 1996. Face encoding and recognition in the human brain. Proc $\mathrm{N}$ atl Acad Sci U SA 93:922-927.

H eckers, S, Rauch, SL, G off, D, Savage, CR, Schacter, D L, Fischman, AJ, Alpert, N M . 1998. Impaired recruitment of the hippocampus during conscious recollection in schizophrenia. $N$ ature $N$ eurosci 1:318-323.

H enke K, Buck A, Weber B, W ieser H G. 1997. Human hippocampus establishes associations in memory. $\mathrm{H}$ ippocampus 7:249-256.

Josephs O, Turner R, Friston K. 1997. Event-related fM RI. H um Brain $M$ ap 5:243-248.

Kapur N , Friston K, Young A, Frith C, Frackowiak R. 1995. Activation of human hippocampal formation during memory for faces: a PET study. Cortex 31:99-108.

Kapur S, Craik FIM , Tulving E, W ilson AA, H ouleS, Brown GM . 1994. N euroanatomical correlates of encoding in episodic memory: Levels of processing effect. Proc N atl Acad Sci U SA 91:2008-2011.

Kapur S, Tulving E, Cabeza R, M clntosh R, H oule S, Craik F. 1996. Neural correlates of intentional learning of verbal materials: a PET study in humans. Cognit Brain Res 4:243-249.

Kelley W, M iezin F, M CD ermott K, Buckner RL, RaichleM E, C ohen N J, Ollinger JM, Akbudak E, Conturo TE, Snyder AZ, Petersen SE. 1998. H emispheric specialization in human dorsal frontal cortex and medial temporal lobe for verbal and nonverbal memory encoding. N euron 20:927-936.

Kopelman M , Stevens T, Foli S, Grasby P. 1998. PET activation of the medial temporal lobein learning. Brain 121:875-887.

Lepage M, H abib, R, Tulving E. 1998. H ippocampal PET activations of memory encoding and retrieval: The HIPER model. Hippocampus 8:313-322.

M aguire $E$, Frackowiak R, Frith C. 1996. Learning to find your way: a role for the human hippocampal formation. Proc R Soc Lond B Biol Sci 263:1745-1750.

M aguire E, Burgess N, Donnett J, Frackowiak R, Frith C, O 'Keefe J. 1998a. Knowing where and getting there: $A$ human navigation network. Science 280:921-924.

M aguire E, Frith C, Burgess N, D onnett J, O 'K eefe J. 1998b. Knowing where things are: Parahippocampal involvement in encoding object locations in virtual large-scale space. J Cognit N eurosci 10:61-76.

M artin A, H axby JV, La Londe FM , Wiggs CL, Ungerleider LG. 1995. Discrete cortical regions associated with knowledge of color and knowledge of action. Science 270:102-105.

$M$ artin A, Wiggs CL, Ungerleider LG, H axby JV. 1996. N eural correlates of category-specific knowledge. N ature 379: 649-652.

$M$ artin A, W iggs CL, Weisberg J. 1997. M odulation of human medial temporal lobe activity by form, meaning, and experience. H ippocampus 7:587-593.

M ontaldi D, M ayes A, Barnes A, Pirie H, H adley D M, Patterson J, Wyper D J. 1998. Associative en coding of pictures activates the medial temporal lobes. Hum Brain M ap 6:85-104.

N yberg L, Tulving E, H abib R, Nilsson L-G, Kapur S, H oule S, Cabeza R, M clntosh AR. 1995. Functional brain maps of retrieval mode and recovery of episodic information. N euroReport 6:249-252.

Nyberg L, M clntosh AR, Houle S, Nilsson L-G, Tulving E. 1996.
Activation of medial temporal structures during episodic memory retrieval. N ature 380:715-717.

O jemann JG, Akbudak E, Snyder A, M cK instry R, Raichle M , Conturo T. 1997. Anatomic localization and quantitative analysis of gradient refocused echo-planar fMRI susceptibility artifacts. Neuroimage 6:156-167.

Ojemann JG, Buckner, RL, Akbudak E, et al. 1998. Functional M RI studies of word stem completion: Reliability across laboratories and comparison to blood flow imaging with PET. Hum Brain Map 6:203-215.

O wen AM , M ilner B, Petrides M , Evans AC. 1996. A specific role for the right parahippocampal gyrus in the retrieval of object location: $A$ positron emission tomography study. J Cognit N eurosci 8:588-602.

Petersson K, Elfgren C, Ingvar M. 1997. A dynamic role of the medial temporal lobe during retrieval of declarative memory in man. N euroimage 6:1-11.

Price CJ, W ise RJS, Watson JD G, Patterson K, H oward D, Frackowiak RSJ. 1994. Brain activity during reading: the effects of exposure duration and task. Brain 117:1255-1269.

Roland P, Gulyás B. 1995. Visual memory, visual imagery, and visual recognition of large field patterns by the human brain: functional anatomy by Positron Emission Tomography. Cereb Cortex 1:79-93.

Rombouts S, M achielsen W, Witter M, Barkhof F, Lindeboom J, Scheltens P. 1997. Visual association encoding activates the medial temporal lobe: A functional magnetic resonance imaging study. H ippocampus 7:594-601.

Rosen B, Buckner R, Dale A. 1998. Event related fM RI: Past, present, and future. Proc N atl Acad Sci U SA 95:773-780.

Roskies AL, Fiez JA, Balota D, Raichle ME, Petersen SE. 1998. $D$ issociable regions in frontal cortex involved in semantic processing (Submitted for publication).

Rudy JW, Sutherland, RJ. 1994. The memory-coherence problem, configural associations, and the hippocampal system. In: Schacter D L, Tulving $E$, editors. M emory systems. C ambridge, M A: M IT Press. $p$ 119-146.

Rugg MD, Fletcher PC, Frith CD, Frackowiak RSJ, Dolan RJ. 1997. Brain regions supporting intentional and incidental memory: a PET study. N euroReport 8:1283-1287.

Schacter DL, Buckner RL. 1998. Priming and the brain. Neuron 20:185-195.

Schacter DL, Reiman E, U ecker A, Polster M R, Yun LS, Cooper LA. 1995. Brain regions associated with retrieval of structurally coherent visual information. N ature 376:587-590.

Schacter DL, Alpert N M, Savage CR, Rauch SL, Albert M S.1996a. Conscious recollection and the human hippocampal formation: Evidence from positron emission tomography. Proc Natl Acad Sci USA 93:321-325.

Schacter D L, Savage CR, Alpert N M , Rauch SL, Albert M S. 1996b. The role of hippocampus and frontal cortex in age-related memory changes: A PET study. N euroReport 7:1165-1169.

Schacter DL, Buckner RL, Koutstaal W, Dale AM, Rosen BR. 1997a. $L$ ate onset of anterior prefrontal activity during retrieval of veridical and illusory memories: A single trial fMRI study. Neuroimage 6:259-269.

Schacter D L, U ecker A, Reiman E, Yun LS, Bandy D, Chen K, Cooper LA, Curran T. 1997b. Effects of size and orientation change on hippocampal activation during episodic recognition: A PET study. N euroReport 8:3993-3998.

Shallice T, Fletcher P, Frith CD, Grasby P, Frackowiak RSJ, D olan RJ. 1994. Brain regions associated with acquisition and retrieval of verbal episodic memory. N ature 368:633-635.

Squire LR. 1994. D eclarativeand nondeclarative memory: M ultiplebrain systems supporting learning and memory. In: Schacter D L, Tulving E, editors. M emory systems. Cambridge, M A: M IT Press. p 203-232.

Squire LR, Ojemann JG, M iezin FM, Petersen SE, Videen TO, Raichle ME. 1992. Activation of the hippocampus in normal humans: $A$ 
functional anatomical study of memory. Proc Natl Acad Sci U SA 89:1837-1841.

Stern CE, Corkin S, G onzalez RG, G uimaraes AR, Baker JR, Jennings PJ, Carr CA, Sugiura RM, Vedantham V, Rosen BR. 1996. The hippocampal formation participates in novel picture encoding: evidence from functional magnetic resonance imaging. Proc $\mathrm{N}$ atl Acad Sci USA 93:8660-8665.

Talairach J, Tournoux P. 1988. C o-planar stereotaxic atlas of the human brain. N ew York: T hieme M edical Publishing.

Taylor SF, Kornblum S, Lauber EJ, M inoshima S, Koeppe RA. 1997. I solation of specific interference processing in the Stroop task: PET activation studies. N euroimage 6:81-92.

Tulving E. 1985. M emory and consciousness. Can Psychol 26:1-12.

Tulving E, Kapur S, M arkowitsch HJ, Craik FIM, H abib R, H oule S. 1994. Neuroanatomical correlates of retrieval in episodic memory: Auditory sentence recognition. Proc N atl Acad Sci USA 91:20122015.

Tulving E, M arkowitsch H, Craik F, Habib R, H oule S. 1996. N ovelty and familiarity activations in PET studies of memory encoding and retrieval. Cereb Cortex 6:71-79.
Ungerleider LG. 1995. Functional brain imaging studies of cortical mechanisms for memory. Science 270:760-775.

Vandenberghe R, Price C, W ise R, Josephs O, Frackowiak R. 1996. Functional anatomy of a common semantic system for words and pictures. Nature 383:254-256.

Wagner AW, Schacter DL, Rotte M, Koutstaal W, M aril A, D ale AM, Rosen BR, Buckner RL. 1998. Building memories: Remembering and forgetting of verbal experiences as predicted by brain activity. Science 281:1188-1190.

Wiggs CL, M artin A. 1998. Properties and mechanisms of perceptual priming: C urr O pin N eurobiol 8:227-233.

W iggs CL, W eisberg J, M artin A. 1999. N eural correlates of semantic and episodic memory retrieval. N europsychologia 37:103-118.

Xiong J, Rao S, Gao J-H, Woldorff M, Fox P. 1998. Evaluation of hemispheric dominance for language using functional $M R I$ : A comparison with positron emission tomography. $\mathrm{H}$ um Brain $\mathrm{M}$ ap 6:42-58.

Zelkowicz BJ, H erbster AN, N ebes RD, M intun M A, Becker JT. 1998. An examination of regional cerebral blood flow during object naming tasks. J Int N europsych Soc 4:160-166. 